\title{
Birth Companions, Health workers perspective: Mixed Method Study in St. Paul's Hospital and Millennium Medical College and its Catchment Centers in Addis Ababa, Ethiopia
}

Kidist Gizachew ( $\sim$ tadelemmakidist@gmail.com )

SPHMMC: St Paul's Hospital Millennium Medical College

Tewodros Getinet

SPHMMC: St Paul's Hospital Millennium Medical College

Delayehu Bekele

SPHMMC: St Paul's Hospital Millennium Medical College

Research

Keywords: Birth companions, health workers, working environment

Posted Date: March 17th, 2021

DOI: https://doi.org/10.21203/rs.3.rs-216483/v2

License: (c) (1) This work is licensed under a Creative Commons Attribution 4.0 International License.

Read Full License 


\section{Abstract}

Background. Birth companions have been shown to improve quality of care provided to laboring women. Various studies have shown the benefits of companion during child birth. The objective of this study was to assess the knowledge, attitude and practice of health care providers towards the involvement of birth companions during child birth in St Paul's Hospital Millennium Medical College (SPHMMC) and its selected catchment health centers.

Methods. The study used a cross-sectional study which was supplemented by qualitative study design which employed phenomenological design was conducted using in depth interview of health care providers who were on practice during the study period in SPHMMC.

Result: The study included a total 58 health care providers. The finding from the study showed that great proportion of health care providers involved in the study (90\%) have positive attitude towards involvement of birth companions but majority $82.4 \%$ didn't allow birth companions. The commonest reasons mentioned for not involving companions include fear of breach of privacy, interference with routine medical care and risk of litigation/complaints.

Conclusion and Recommendation: The findings from this study showed that majority of the health care providers are against the practice of involving birth companions. Since involvement of birth companions is one way to ensure the quality of care provided there should be an effort to increase the knowledge of health professionals on benefits of birth companions. There is also a need for improvement of the work setup. Simple measures like providing screens, providing separate rooms for laboring and post-partum women will have a significant impact on the quality of care provided.

\section{Background}

Birth companion has been mentioned by the WHO as one of the most cost effective methods to increase positive child birth experience.[1]Various randomized controlled trials have shown that the involvement of birth companion is associated with better maternal and neonatal outcome.[2,3] There are also evidences that verify the positive perception and trust women had on birth companions. Essentially, this emanates from the strong belief of women that the quality of care they received is improved when they have birth companion. $[4,5]$

A study done in SPHMMC and three health centers in Addis Ababa assessed the level of respect and nonabusive care accorded to women delivering in health facilities.

The findings of this study showed that all women who delivered in the hospital and $89.4 \%$ of women who delivered in the health centers experience the violation of right to information, lack of informed consent, and denied of the opportunity to deliver in birth position of their choice.[6]Although this study assessed the various aspects of disrespect and abuse it did not explore the extent of involvement of birth companions claiming the service as something that lack in most setups in the country. The investigators 
recommended the need to promote women-centered care as this was believed to positively influence women's decision to deliver at health facilities.

In countries where the health delivery systems are well developed and facilitated, maternal mortality and morbidity substantially decreased and the perinatal outcome improved appreciably. The progresses made in these fronts are minimal in developing countries. According to EDHS (2016), the current institutional delivery rate in Ethiopia is $26 \%$. [7]This is irrespective of the quality of care and satisfaction level provided to women who used the health delivery system. Reportedly, there are cases where the quality of the services is regarded by postpartum mothers or their families less satisfactory. Researches show that women who are dissatisfied with the care they receive from health facilities tend to resort to traditional system. [8, 9] A cross sectional study conducted by the University of Gondar showed that only $31 \%$ of mothers' who delivered in this facility were satisfied with the care they received.[10]

This suggests the need that health facilities had to work hard to improve the quality of care they provide and hence improve maternal satisfaction. Number of interventions are available for the formal health delivery system in order to meaningfully improve the maternal satisfaction and the neonatal outcomes. One of these interventions is involvement of birth companion.

Detailed and empirical studies exploring the perception of health professionals and women towards birth companions within the Ethiopian health facilities is either totally lacking or at best is limited in scope. It is speculated that the reason for limited involvement of birth companions might be related to socio-cultural barriers and to the limited awareness of its benefits among health practitioners and child expecting women.

This study is done in two phases wherein the first one assessed the health workers knowledge, attitude and practice with regards to birth companions. This was followed by a second study which assessed post-partum women's perception with regards to involvement of birth companions. The study explored the extent of involvement of birth companions and the factors that restricted the implementation of the practice in labor wards. This part of the manuscript presents the finding from the first of the phase of the study.

The objectives for this study were;

- To determine the level of knowledge on benefits of birth companions among health care providers in SPHMMC and selected catchment health centers

- To find out the attitude of health care providers at SPHMMC and its selected catchment health centers on involvement of birth companions

- To find out the current practice and factors that hinder routine inclusion of birth companions in SPHMMC and its catchment health centers

\section{Methods}


Study area and period

The study was conducted at SPHMMC and its catchment health centers in Addis Ababa. The hospital, which serves as referral health establishment provides delivery service to over 10,000 mothers in a year. On average, the number of monthly deliveries stands at about 1000 . This figure does not include deliveries in the 16 catchment health centers. These centers have a well-organized referral and feedback system with the hospital.

The Obstetrics and gynecology department of SPHMMC has around 120 residents and more than 20 obstetricians. It has more than 90 midwives. The obstetrics department is regularly visited by expatriate obstetricians and has basic facilities that help handle routine and complicated delivery cases.

This study mainly targeted health professionals working at the labor ward of SPHMMC and the selected catchment health centers. The study was conducted from September to December 2019.

Study design

The study used mixed method study which used quantitative cross-sectional study which was supplemented by qualitative study. The qualitative study used phenomenological design. It used thematic analysis where themes were developed after collection of the data.

Study population

The study populations of this study are health professionals that work in SPHMMC and its catchment health centers. These professionals include obstetrics and gynecology specialists, residents, and midwives who are currently on practice for more than 6 months in these institutions.

Sample size and sampling method

The health professionals included in the study were all health care providers who were involved in the day today activities of the labor ward during the study period in SPHMMC and in the randomly selected catchment heath centers during the study period.

The data for the qualitative study was collected among 7 health care providers. This was collected using in-depth interviews as a study tool, which was conducted among health care providers who are involved in the delivery service at SPHMMC.

\section{Data collection}

Data with regards to the perception and practice of health care providers towards the involvement of birth companions in labor wards was collected using self-administered structured questionnaires. Midwives and obstetricians with more than 6 months of experience in the health care establishments and residents of second year and above were included. The questionnaire is adopted from a similar study that was conducted in Sri Lanka. [11] 
In-depth interview was undertaken by the principal investigator, KG, among 3 midwives and 4 residents who were working in the labor ward during the study period. The interviewer used interview guide. The participants were chosen based on the number of years they served in the institution. The discussions were tape recorded.

\section{Data analysis}

Once the data gathering is completed, it was then entered into Epidata and cleaned and analyzed using SPSS Version 20.0. Determination of the frequency of the socio demographic factors and practice with regards to involvement of birth companions was conducted.

The results from the in-depth interview were recorded and transcribed verbatim in the local language (Amharic) then translated into English. A coding scheme was developed by the principal investigator. Open code software was used to assist the coding process. Thematic analysis method was used for analysis of the qualitative data.

\section{Ethical consideration}

Ethical approval was secured from the ethical review board of SPHMMC before the start of the study. Support letter was prepared and submitted to heads of selected health centers. Prior to the collection of the study data, consent was obtained from study participants. Study participants were not required to mention their names and participation in the study was on volunteer basis.

Definition of terms used in the study

These are defined after thorough review of the literatures. [2, 12]

\section{Birth companion}

anyone who accompanies a laboring woman during the birth process

\section{Doula}

a trained lay person who accompanies a laboring woman during the birth process

\section{Knowledgeable on the benefit of birth companions}

Those health care providers who mentioned at least three benefits of birth companions

\section{Results}

The cross-sectional study included a total of 51 health care providers who were providing obstetrics and gynecology service during the study period. These were 8 obstetrics and gynecology specialists, 26 residents and 17 midwives. 
The mean age of the health workers included in the study was 30 years with a SD of 3.6. Married study participants include $58.8 \%$ of the health care providers. The longest years in obstetric practice is 15 years while the least amount of time on the health care service specifically in the delivery service is 2 years. (Table 1)

Table 1

Socio-demographic characteristics of health care providers included in the study at SPHMMC and selected catchment health centers

\begin{tabular}{|lll|}
\hline & Total number & Percentage \\
\hline Marital Status & & \\
\hline Married & 30 & 58.8 \\
\hline Single & 21 & 41.2 \\
\hline Religion & & \\
\hline Orthodox Christian & 37 & 72.5 \\
\hline Protestant & 9 & 17.6 \\
\hline Islam & 5 & 9.8 \\
\hline Profession & & \\
\hline Midwife & 17 & 33.3 \\
\hline Obstetrics and gynecology resident & 26 & 51 \\
\hline Obstetrics and gynecology senior & 8 & 15.7 \\
\hline
\end{tabular}

Knowledge, Attitude and Prevailing practice with regards to birth companions

Among the health care providers involved in the study $39.2 \%$ were knowledgeable on benefits of birth companions.

Among the study participants $90 \%$ has positive attitude towards involvement of birth companions while only $4 \%$ has negative attitude.

From the health practitioners involved in the study $82.4 \%$ didn't allow labor companions. The reasons that were mentioned include lack of adequate space, busy labor ward, lack of partitioning among labor ward beds and doubt on the benefit of birth companions. (Table 2) 
Table 2

Reasons for not allowing birth companions mentioned by health care providers involved in the study

\begin{tabular}{|lll|}
\hline Reasons for not allowing birth companions & Total number & Percentage \\
\hline Lack of adequate space & 32 & 62.75 \\
\hline Busy labor ward & 25 & 49.02 \\
\hline Absence of partitioning at the labor ward & 12 & 23.53 \\
\hline Doubt on the benefit of birth companions & 2 & 3.92 \\
\hline Society don't agree with the practice & 3 & 5.88 \\
\hline
\end{tabular}

With regards to benefits of birth companions $96.1 \%$ of the health care providers believe that there is benefit in their involvement. The benefits mentioned were improved moral support to mothers, improved care by health workers, advantages in infant attachment, reduction of interventions, shortened length of labor, ease of communication with mothers, improved trust of health professionals, reduced home deliveries and decreased maternal stress. (Table 3 )

Table 3

Benefits of birth companions mentioned by health care providers involved in the study

\begin{tabular}{|lll|}
\hline Benefits of birth companions & Total number of health care providers & Percentage \\
\hline Moral support to mothers & 47.0 & 92.16 \\
\hline Improves care by health care providers & 16.0 & $31 . .37$ \\
\hline Facilitate breast feeding & 28 & 54.90 \\
\hline Reduces operative deliveries & 13 & 25.49 \\
\hline Shortens duration of labor & 22 & 43.18 \\
\hline Reduces the need for augmentation & 11 & 21.57 \\
\hline Improves neonatal outcome & 9 & 17.65 \\
\hline Ease of discussion with laboring mothers & 2 & 3.92 \\
\hline Reduces home deliveries & 4 & 7.84 \\
\hline Decrease labor pain & 1.0 & 1.96 \\
\hline
\end{tabular}

The health care providers who were included in the study mentioned that they have faced breach of privacy, risk of litigation/complaints, interference with routine medical care, risk of theft and worsened perception of health care providers by involving birth companions. (Table 4) 
Table 4

Disadvantages of birth companions mentioned by health workers in the study

\begin{tabular}{|lll|}
\hline Disadvantage & Total number & Percentage \\
\hline Breach of privacy & 23 & 45.09 \\
\hline Possibility of litigation & 13 & 25.49 \\
\hline Interference with routine medical care & 22 & 43.14 \\
\hline Risk of theft & 4 & 7.84 \\
\hline Worsen distrust of health professionals & 1 & 1.96 \\
\hline
\end{tabular}

\section{Health workers Perspective on involvement of birth companions: Findings from in-depth interview of health workers}

In- depth interview of seven health professionals was undertaken to further understand the attitude of health care providers with regards to birth companions.

\section{Prevailing practice while managing laboring women}

The finding from the study has showed that every laboring woman that comes to the hospital goes through the emergency gynecology team. She will be evaluated initially by midwife, intern or junior resident. After that depending on the urgency of the case the most senior person from the managing team will evaluate her.

After evaluation the managing team will send baseline investigations and until bed is found the woman will be kept either inside the ER or in the corridors. And the team will prioritize among the laboring women's in the ER and decide who will be admitted.

During a woman's stay in the emergency she will usually be allowed to be around her family members, outside the emergency room. She will intermittently be called and evaluated. If she has any indication to be kept inside the whole time, no family member will be allowed to see her.

Once a patient is admitted to the labor ward a midwife or a resident will accept her. At the labor ward she will be evaluated and someone will be assigned to follow her. Depending on the stage of labor, condition of the laboring woman, status of the labor ward, the follow-up intensity will vary. Usually there will be intermittent follow-up of the laboring woman. There are times the woman will be left alone.

According to one resident 'There is no one single person who will continuously be with her, a midwife, an intern or a resident will follow her but they have some tasks in between so there are a lot of times she will be left alone. If it is spontaneous labor and if she doesn't have any risk it is only the CTG who will be with 
her. People will come in see the CTG and move on. There are also times we listen to the CTG while we are in the next room'.

\section{Practice on involvement of labor companions}

Health workers explained that in SPHMMC birth companions are always family members, this ranges from husband, sisters, mothers, brothers or friends and neighbors. The word doula is strange for almost all of them.

Results from the interviews show that most of the health care providers don't allow birth companions; this applies both to labor ward and emergency room. They have explained whatever their opinion they follow the mass in not allowing birth companions. Those who allow companions described they allow them to intermittently see their loved ones and give update to the family members on how the labor is progressing and if there is a need for further intervention.

'On principle there will not be a companion with the laboring woman" final year resident with around six years experience in SPHMMC.

'But I am also not different. I rarely update the family and won't let family members inside' one final year resident explained.

Birth companions are involved in few circumstances. Those women who have eclampsia or any condition that deserves frequent follow up are the ones who will be allowed to have companions. One of the residents explained 'In SPHMMC there has never been a birth companions, unless the woman has ecclampsia or something like that and we want someone to restrain or hold a woman, we never allow husbands or anyone for that matter. And the reason is the setup we have, there will be two or more laboring mothers in one place and to allow attendants it will breach other women's privacy.'

Post-partum period is the one time where majority of the women will be allowed to have companions around.

For the majority of the women who didn't get to see anyone whoever is following them will go and get them what they need and pass messages to and from the companions since phones aren't allowed. But there are times the companions wouldn't hear what has happened to the laboring women.

Every one of the health care providers have different reasons for not involving birth companions. But there are reasons that are mentioned by most of the health care providers. These include need for privacy, work load, case load, set-up, knowledge and comfort of health care providers for providing care to laboring women while companions are around.

\section{Opinion on involvement of birth companions}

There is Ethiopian ministry of health's recommendation on involvement of birth companions. It states birth companions should be allowed to be with laboring women. While asked what is their perception to 
this recommendation most health care providers explained though they agree with the principle they don't think it is applicable to the set-up they work in.

'Of course, I think involvement of birth companions is important' a midwife with over two years experience in SPHMMC.

Benefits and difficulties they faced with involvement of labor companions

Even those who didn't involve birth companions agreed that there is a benefit in companion involvement. The benefits they mentioned were ease of communication, relief of pain, benefit on attachment and for the mothers to attain birth positioning of choice.

The difficulties mentioned by health care providers during involvement of companions include argument with health workers, frequent nagging, risk of litigation and lack of awareness on the ongoing care the woman is getting and interference due to this.

The main cause of argument between the health care providers and the companions is the need to be with the laboring woman throughout the labor process. There were situations mentioned by health care providers that have escalated into fights.

One health care provider mentioned his experience; "once I have seen a companion who hit a midwife. This is because the health professional was telling the companion to go out, he wanted to evaluate the woman who was sharing the same room with the woman this companion was with, but he refused and it turned into a heated debate. This went on for a while and the companion hit the midwife..."

\section{Reasons for not allowing labor companions}

The main setback mentioned for involvement of birth companions is the issue of privacy. The participants mentioned that there will be multiple laboring mothers next to each other with-out proper screen. This makes allowing companion of one mother difficult. According to one health care provider "There are women who only has male companions; there are Muslim mothers who don't want to be seen by males; so for the sake of one laboring mother we shouldn't trespass the right of others. Even if they have female companions it is not fair to be seen by someone you don't know."

The other commonly mentioned problem is the existing setup. This includes the presence of laboring rooms without screens, lack of adequate space to add companions, lack of adequate couches and laboring beds. And one health worker explained his stand by saying: "with regards to birth companions I follow the mass, I don't allow because of the setup we have".

The case load is also one hindrance to involvement of birth companions. At SPHMMC there will be a minimum of 800 deliveries per month and the ER will be crowded with laboring mothers which makes even proper evaluation of patients challenging. The health workers mentioned that there are a lot of laboring mothers at specific time at the ER and addition of companions becomes a luxury. 
Other factors include fear of litigation if any complication arouse and lack of adequate knowledge on the benefit of birth companions. There are also health care providers who mentioned they won't feel comfortable evaluating a laboring woman with family members nearby.

There was conflicting finding with regards to the understanding on the administration stand on the issue of birth companions. There is a $\mathrm{MOH}$ recommendation and the institutions commitment to abide with that recommendation. But there is also institutions rule to allow companions only in the morning and late in the afternoons. The hospital has hired guards which make sure family members are allowed only on the allocated time period. This rule is implemented in the labor wards also. And the restrictions are more stringent at labor ward.

\section{Discussion}

The finding from our study has shown among the health care providers who were involved on the study $39.2 \% \%$ were knowledgeable of this benefits. There are various studies which showed the multiple benefits of birth companions. A Cochrane systematic review which included fifteen trials involving 12,791 women showed that women who had continuous intrapartum support were less likely to have intrapartum analgesia, operative birth, or have dissatisfaction with their childbirth experiences. [2] The benefit of birth companions was also emphasized by WHO.[1]

Federal ministry of health of Ethiopia has prepared a guideline addressing the issue of compassionate, respectful and caring maternity service. And according to this recommendation all laboring women should be accompanied by birth companion of their choice. Though, the possibility of implementing this recommendation in the existing system is clearly challenging, the finding from our study has showed the prevailing practice is far from the ministries recommendation.

In this study $96.1 \%$ of the health care providers believe that there is benefit of birth companions. This finding is comparable to findings of researches done in other African countries. [5, 13]From these a qualitative study done in Zambia explored the views of 84 mothers and 40 health staff about the involvement of birth companions. In this study, most of the mothers appreciated the emotional and practical support of birth companions and a good number of the health care provider believe that the social support will give the women sense of security and help the laboring women.

From the health practitioners involved in this study $82.4 \%$ didn't allow labor companions. A similar finding was made by a study done in Kenya. Similarly increased involvement of birth companions when the mother is difficult to deal with is also seen [16]. The comparable socio-economic conditions of the two nations might have contributed to the similarity.

The commonest reasons mentioned by health care providers for not involving birth companions in this study include breach of privacy, interference with routine labor ward activity, lack of space and the volume of work in the labor wards among others. Other studies have also shown similar factors affecting implementation. Among this a systematic review that tried to assess the factors affecting 
implementation of birth companions has shown that health care providers were concerned about the role of birth companions and their interference with labor ward activities. The reviewers have reached to a conclusion of the need to sensitize health care providers on the issue and the need to study factors affecting implementation in different setups. [15]A Cochrane qualitative synthesis has also shown the factors affecting implementation include lack of knowledge among women and health care providers and lack of space [16].

Another study done to understand the feasibility of engaging birth companions within the health facilities of developing countries, showed $58.8 \%$ of obstetricians did not allow labor companions in their wards [11]. The reasons mentioned for this practice were similar to the hindrances described in our study.

\section{Conclusion}

The findings from our study showed that labor companions are not part of the routine practice in SPHMMC and its catchment health centers. Though limited in depth majority of health professionals have knowledge on the benefit of birth companions. The commonest reasons mentioned by health care providers not to involve companions include lack of adequate space and difficulty to ensure privacy.

\section{Recommendation}

Involvement of birth companions is one way to ensure the quality of service provided efforts should be made to increase the knowledge of health professionals on benefits of birth companions and also to address the setbacks faced to involve companions. There is also a need for improvement of the work setup. This includes simple measures like providing screens, providing separate rooms for laboring and post-partum women.

\section{List Of Abbreviations}

ANC- Antenatal care

EDHS- Ethiopian demographic and health survey

ETB- Ethiopian Birr

SPHMMC- St. Paul's Hospital Millennium Medical College

WHO- World Health Organization

\section{Declarations}

Ethical approval and consent to participate 
Ethical approval was secured from the ethical review board of SPHMMC before the start of the study. Support letter was prepared and submitted to heads of selected health centers. Prior to the collection of the study data, consent was obtained from study participants. Study participants were not required to mention their names and participation in the study is on volunteer basis. Minors weren't included in the study.

Consent for publication

No information that disrupts anonymity of the study participants was included.

Availability of data and materials

All data used to generate the finding of the study are available from the corresponding author upon request.

Competing interest

The authors declare that they have no competing interest.

Funding

Fund for the research was obtained from St. Paul's hospital millennium medical college. Despite providing fund for the study the institution was not involved in design of the study, data collection, analysis or interpretation of the findings of the study.

Author's contribution

KG helped in proposal writing, data analysis and write-up of the finding of the study. DB and TG have participated in development of study design and write-up of the findings of the study. All authors read and approved the final manuscript.

Acknowledgment

The authors would like to thank the St Paul's Hospital Millennium Medical College (SPHMMC) for funding the research work within the College and its affiliate health facilities. The authors would like to thank Dr. Ewenet for her invaluable insight and continuous support.

\section{References}

1. Tuncalp Ó!, Were WM, MacLennan C, Oladapo OT, Gulmezoglu AM, Bahl R, Daelmans B, Mathai M, Say L, Kristensen F, Temmerman M, Bustreo F. Quality of care for pregnant women and newbornsthe WHO vision. BJOG. 2015;22:10459. http://onlinelibrary.wiley.com/doi/10.1111/1471$0528.13451 / p d f$. 
2. Hodnett ED, Gates S, Hofmeyr, GJand C.Sakala. 2011. Continuous Support for Women during Childbirth. Cochrane Database SysRev: 7:CD003766.doi:1002/14651858.CD003766. pub5.

3. Marshall h Klaus, john h. Kennell. Steven s Robertson, Roberto Sosa.1986. Effects of social support during parturition on maternal and infant morbidity. British medical journal 293:585-588.

4. Tamar Kabakian-khasholian, Bashour AEI-Nemer,H. Perception about labor companionship at public teaching hospitals in three Arab countries. International journal of gynecology obstetrics. 2015;129:3:223-6. http://doi.org/10.1016/j.ijgo.2014.12.005/pdf.

5. Banda G, Kafulafula E, Nyirenda F, Taulo LK.2010. Acceptability and experience of supportive companionship during childbirth in Malawi BJOG 117:937-45.DOI:10.1111/j.14710528.2010.02574.x. .

6. Anteneh Asefa and Delayehu Bekele. 2015. Status of respectful and non-abusive care during facility based childbirth in a hospital and health centers in Addis Ababa, Ethiopia. Reproductive health 12:33. http.//doi 10.1186/s12978-015-0024-9.

7. Ethiopia. Demographic and Health Survey.2016. CSA.

8. Dzomeku MV. Maternal satisfaction with care during labor: A case study of the Mampong- Ashanti district hospital maternity unit in Ghana. International journal of Nursing midwifery. 2011;3:30-4.

9. Redshaw M, Hikkila K.2010.Delivered with care: a national survey of women's experience of maternity care USA. The national perinatal epidemiology unit, University of oxford, old road campus, oxford ox37LF. 61 p.

10. Gashaye KT, Tsegaye AT, Shiferaw G, Worku AG. Solomon Mekonen Abebe. 2019. Client Satisfaction with the existing labor and delivery care and associated factors among mothers who gave birth in University of Gondar teaching hospital; Northwest Ethiopia: Institution based cross-sectional study. Open Access. http://doi.org/10/journal.pone.0210693.

11. Hemantha, Senanayake, Rajitha Dilhan Wijesinghe and Kesavan Rajasekharan Nayar. 2017. Is the policy of allowing a female labor companion feasible in developing countries? Results from a cross sectional study among Sri Lankan practitioners. BMC Pregnancy Childbirth 17:392.http.//doi 10.1186/s12884-017-1578-z.

12. Microsoft. Encarta Dictionary. Doula.

13. Margaret C. Maimbolwa.2019. Views on involving a social support person during labor in Zambian maternities. Journal of midwifery and women's health 64:2.http.//onlinelibrary.wiley.com/doi/full/10.1016/pdf.

14. Tamar Kabakian-Khasholian and Anayda Portela.2017. Companion of choice at birth: factors affecting implementation. BMC Pregnancy Childbirth 17:265. http.//link.springer.com/article/10.1186/s12884-017-1447-9.

15. Perceptions and experiences of labour companionship: a qualitative evidence synthesis. Cochrane database of systematic review. 2019, Issue 3. Art. No.: CD012449.

16. Patience Afulani C, Kusi, Leah Kirumbi and Dilys Walker. Companionship during facility-based childbirth: results from a mixed-methods study with recently delivered women and providers in 
Kenya. BMC Pregnancy childvirht. 2018;18:150.

\section{Supplementary Files}

This is a list of supplementary files associated with this preprint. Click to download.

- Corecchecklist.docx 